\title{
Fermion loop simulations in 2-d lattice theories - results and limitations
}

\author{
Markus Limmer* \\ Institut für Physik, FB Theoretische Physik, Universität Graz, 8010 Graz, Austria \\ E-mail: markus.limmer@uni-graz.at
}

\section{Christof Gattringer}

Institut für Physik, FB Theoretische Physik, Universität Graz, 8010 Graz, Austria

E-mail: christof.gattringer@uni-graz.at

\section{Verena Hermann}

Department of Earth and Environmental Sciences, Geophysics, Munich University, 80333

Munich, Germany

E-mail: verena.hermann@geophysik.uni-muenchen.de

We review our results for the simulation of the $2 \mathrm{~d}$ lattice Gross-Neveu model in a fermion loop representation. Possible extensions of our techniques to other models and higher dimensions are discussed, as well as the limitations of loop-type representations.

The XXV International Symposium on Lattice Field Theory

July 30 - August 4, 2007

Regensburg, Germany

${ }^{*}$ Speaker. 


\section{Introduction}

Numerical simulations of fermion systems have to deal with the Pauli principle which enforces a completely anti-symmetric wave function for fermions. Obviously, this requirement makes fermion systems extremely non-local. Changing the system at a single point affects all degrees of freedom. In more technical terms, an ad-hoc local change may lead to a completely different value of the fermion determinant. Thus intricate methods such as the hybrid Monte Carlo algorithm were developed. Nevertheless, also these methods have to deal with the non-locality which makes the simulation of fermions several orders of magnitude more expensive than bosonic systems.

Circumventing the non-locality problem altogether is certainly an extremely appealing idea. A prominent example of a breakthrough in this direction is the Meron Cluster Algorithm proposed in [1], which allows for highly effective Monte Carlo simulation for certain classes of fermionic models.

An alternative approach is a formal solution of the Grassmann path integral for fermions which represents the partition function of the system as a model of closed fermion loops. While for a long time this technique has been known to work well in the strong coupling limit, only recently $[2,3]$ loop representations were found for two-dimensional lattice field theories at arbitrary coupling, in particular the Gross-Neveu model [4].

In a recent publication [5] it was shown that the loop representation allows for an efficient and considerably cheaper simulation than traditional methods. In a subsequent paper [6] Wolff has rederived the loop representation by decomposing 2- $\mathrm{d}$ Dirac fermions into Majorana components and demonstrated that the loop formulation can be recast as a spin system where a cluster algorithm boosts the efficiency of a numerical simulation further.

In this contribution we review the loop representation of the lattice Gross-Neveu model and its use for a numerical simulation. We furthermore discuss the loop representation of the Schwinger Model [7], i.e., QED in two dimensions, an example which illustrates the limitations of the loop approach for a use in numerical simulations.

\section{Loop representation of the lattice Gross-Neveu model}

We consider the lattice Gross-Neveu model with $N$ flavors of Wilson fermions. The lattice action for the fermions is given by (we set the lattice spacing to $a=1$ )

$$
\begin{aligned}
S_{F}[\bar{\psi}, \psi, \varphi] & =\sum_{x \in \Lambda} \bar{\psi}(x) D(x, y) \psi(y), \\
D(x, y) & =[2+m+\varphi(x)] \delta_{x, y}-\sum_{\mu= \pm 1}^{ \pm 2} \frac{1 \mp \gamma_{\mu}}{2} \delta_{x+\hat{\mu}, y} .
\end{aligned}
$$

The sum runs over the $2-\mathrm{d}$ lattice $\Lambda$. In two dimensions the $\gamma$-matrices may be chosen as the Pauli matrices, $\gamma_{ \pm \mu}=\sigma_{\mu}$. The spinors $\bar{\psi}$ and $\psi$ are vectors of $N 2-\mathrm{d}$ spinors, and we use vector/matrix notation for both the spinor and flavor indices. Through the Dirac operator $D(x, y)$ all flavors couple in the same way to the real scalar field $\varphi$, which has the action

$$
S_{S}[\varphi]=\frac{1}{2 g} \sum_{x \in \Lambda} \varphi(x)^{2} .
$$


When integrating out the scalar field the 4-fermi interaction

$$
-\frac{g}{2} \sum_{x \in \Lambda}(\bar{\psi}(x) \psi(x))^{2}
$$

is induced. The partition function of the model is given by

$$
Z=\int \prod_{x} d \varphi(x) d \bar{\psi}(x) d \psi(x) e^{-S_{S}[\varphi]-S_{F}[\bar{\psi}, \psi, \varphi]}=\int \prod_{x} d \varphi(x) e^{-S_{S}[\varphi]} \operatorname{det}(D[\varphi])^{N},
$$

where in the second step the fermions were integrated out giving rise to a remaining path integral over the scalar field with the fermion determinant raised to the power $N$ as integrand.

The last expression is a possible starting point for identifying the loop representation. The Dirac operator $D$ may be rewritten as

$$
\begin{aligned}
& D(x, y)=[2+m+\varphi(x)]\left[\delta_{x, y}-H(x, y)\right], \\
& H(x, y)=\frac{1}{2+m+\varphi(x)} \sum_{\mu= \pm 1}^{ \pm 2} \frac{1 \mp \gamma_{\mu}}{2} \delta_{x+\hat{\mu}, y},
\end{aligned}
$$

where we have combined all nearest neighbor terms in the hopping matrix $H$. Inserting the representation (2.5) into the partition function (2.4) one finds

$$
\begin{aligned}
Z & =\int \prod_{x} d \phi(x) e^{-S_{S}[\varphi]} \prod_{x}(2+m+\varphi(x))^{2 N} \operatorname{det}(1-H[\varphi])^{N} \\
& =\int \prod_{x} d \phi(x) e^{-S_{S}[\varphi]} \prod_{x}(2+m+\varphi(x))^{2 N}\left(\exp \left(-\sum_{n=1}^{\infty} \frac{1}{n} \operatorname{Tr}\left[H^{n}\right]\right)\right)^{N},
\end{aligned}
$$

where we have used the formula $\operatorname{det}[1-H]=\exp (\operatorname{Tr} \ln [1-H])$ for the determinant and expanded the logarithm.

The expression (2.6) is the well known hopping expansion. At this point the loops are already evident: The hopping matrix $H$ is a matrix which describes hopping between neighboring lattice points. Consequently the power $H^{n}$ in (2.6) corresponds to a chain of $n$ subsequent steps. When taking the trace only closed chains, i.e., loops survive. Such an expansion holds in arbitrary dimensions and for different types of bosonic fields, scalar, as well as gauge fields.

The crucial step, however, is that the traces $\operatorname{Tr}\left[H^{n}\right]$ in (2.6) can be evaluated only in special cases. In addition to the space-time indices, this trace is over Dirac and for non-abelian gauge theories also over the color indices. For the latter a simple closed form is probably not realistic. Concerning the Dirac indices, in two dimensions it is possible [8] to find a closed form for the trace over the matrices $\left[1 \pm \gamma_{\mu}\right] / 2$ which enter the hopping matrix (2.5). Thus for the case of non-abelian interactions in two dimensions the exponent in (2.6) can be computed in closed form.

As discussed, for special cases (scalar or abelian bosonic fields in 2-d) the coefficients for the individual loops in the exponent of (2.6) can be computed analytically. The final step is to bring the loops down from the exponent. Here two different approaches were followed in [3] and [7]. In the former case the final expression for the loop representation was obtained by comparing the 2-d Wilson fermions to the hopping expansion of a 8-vertex model. In the latter case a direct evaluation of the exponential of the sum over loops was performed. Again we remark that there is also 
the direct identification of the loop representation through the explicit solution of the Grassmann integral for the Majorana components [6].

Once the determinant is given as a sum over loops with known coefficients (not as the exponential of a sum over loops) there is only the path integration over the bosonic variables attached to the loops left to be done. For the case of the scalar fields which give rise to the 4-fermi interaction, the path integration is trivial, since at each lattice point only moments of the Gaussian distribution need to be computed. In this way the partition function of the $N$-flavor lattice Gross-Neveu model is found to be a model of $2 N$ self-avoiding loops. For the case of general $N$ we refer the reader to [2], and here quote the result for $N=1$, which is the case that was used in the numerical simulations $[5,6]$. The partition function reads

$$
Z=\sum_{r, b}\left(\frac{1}{\sqrt{2}}\right)^{c(r, b)} f_{1}^{n_{1}(r, b)} f_{2}^{n_{2}(r, b)} .
$$

The sum runs over two sets of loops which we refer to as red $(r)$ and blue $(b)$. For a given color the loops are self avoiding, i.e., they cannot cross or touch each other, while loops of different may do so. In Eq. (2.7) $c(r, b)$ is the total number of corners for both, red and blue loops. Thus every corner contributes a factor of $1 / \sqrt{2}$ to the weight of a configuration. Furthermore, $n_{1}(r, b)$ is the number of lattice sites which are singly occupied by either $r$ or $b$ and $n_{2}(r, b)$ is the number of doubly occupied sites, i.e., sites which are visited by both, a red and a blue loop. The weight factors $f_{1}$ and $f_{2}$ are simple functions, related to the mass $m$ and the coupling $g$ through

$$
f_{1}=\frac{2+m}{(2+m)^{2}+g} \quad, \quad f_{2}=\frac{1}{(2+m)^{2}+g} .
$$

The mapping (2.7), (2.8) is exact in the thermodynamic limit. For finite volume different types of boundary conditions in the two representations lead to finite size effects: In the loop representation we need to have closed loops and in a finite volume the loops can wind around the periodic boundary. The loop configurations fall into three equivalence classes, $C^{e e}, C^{e o}, C^{o o}$, depending on the numbers of red and blue non-trivially winding loops (see also [9]): $C^{e e}$ (even-even): The total number of windings for both, red and blue loops is even for both directions. $C^{e o}$ (even-odd): One of the colors has an odd number of windings for one of the directions. $C^{o o}$ (odd-odd): Both colors have an odd number of windings in one of the directions. These equivalence classes cannot be linked in a simple way to the boundary conditions in the standard representation which we discussed above. However, in [5] it was shown that the boundary effects vanish as $1 / \sqrt{V}$, with $V$ denoting the volume. The representation in terms of the Ising spin variables [6] solves the boundary condition problem completely, and the partition functions of the original fermionic- and the spin representation are identical also on finite volumes.

\section{Numerical simulation}

For the numerical simulation of the loop representation of the Gross-Neveu model we use a local Metropolis update. Red and blue loops are updated alternately by performing a full sweep through the lattice for only one color and meanwhile treating the other as a constant background field. During one sweep all plaquettes are visited once. A trial configuration is offered by inverting 



Figure 1: Typical loop configurations in the 1-flavor Gross-Neveu model. We compare two different values of the parameters. L.h.s.: $g=0.0, m=0.1$; r.h.s.: $g=0.0, m=0.2$.

the 4 links of the current plaquette of the active color. With such an offer, we guarantee that the loops stay closed, or new loops are created if all links of the plaquette were empty before. In case that the self-avoidance condition is violated, the proposal is rejected. Otherwise the new configuration is accepted with the Metropolis probability

$$
p=\left(\frac{1}{\sqrt{2}}\right)^{\Delta c} f_{1}^{\Delta n_{1}} f_{1}^{\Delta n_{1}}
$$

$\Delta c$ is the difference of the number of corners, $\Delta n_{1}$ and $\Delta n_{2}$ are the differences in the occupation numbers. Fig. 1 shows snapshots of typical loop configurations in the numerical simulation.

Particularly simple observables are derivatives of the free energy $F=-\ln Z$. These expressions can be written as moments of occupation numbers. To be more explicit we discuss the chiral condensate $\chi$ and its susceptibility $C_{\chi}$. The conventional definitions are

$$
\begin{aligned}
\chi & =\frac{1}{V} \sum_{x \in \Lambda}\langle\bar{\psi}(x) \psi(x)\rangle=-\frac{1}{V} \frac{\partial \ln Z}{\partial m}, \\
C_{\chi} & =\frac{\partial \chi}{\partial m} .
\end{aligned}
$$

In terms of loop variables these expressions read

$$
\begin{aligned}
\chi= & -\frac{1}{V f_{1}}\left[f_{2}\left\langle n_{1}\right\rangle+2 f_{1}^{2}\left\langle n_{0}\right\rangle\right], \\
C_{\chi}= & -\frac{1}{V f_{1}^{2}}\left[\left(4 f_{1}^{4}-2 f_{1}^{2} f_{2}\right)\left\langle\left(n_{0}-\left\langle n_{0}\right\rangle\right)^{2}\right\rangle+\left(f_{2}^{2}-2 f_{1}^{2} f_{2}\right)\left\langle\left(n_{1}-\left\langle n_{1}\right\rangle\right)^{2}\right\rangle\right. \\
& \left.+2 f_{1}^{2} f_{2}\left\langle\left(n_{0}+n_{1}-\left\langle n_{0}+n_{1}\right\rangle\right)^{2}\right\rangle-\left(4 f_{1}^{4}-2 f_{1}^{2} f_{2}\right)\left\langle n_{0}\right\rangle-f_{2}^{2}\left\langle n_{1}\right\rangle\right],
\end{aligned}
$$



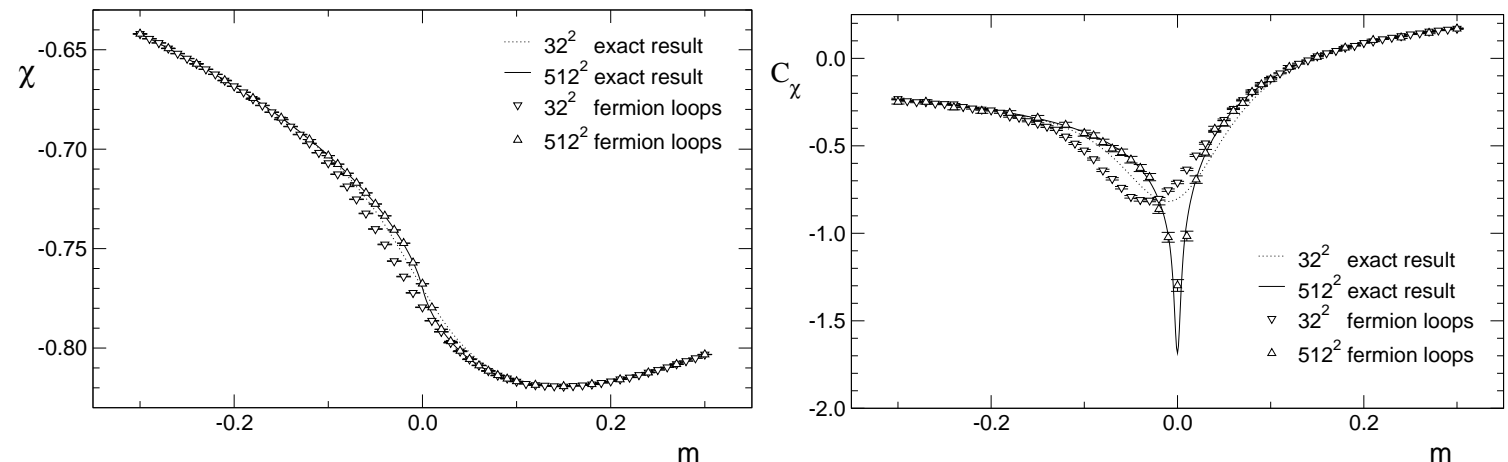

Figure 2: L.h.s.: The chiral condensate $\chi$ for $g=0$ as a function of $m$ for 2 different lattice sizes. We compare the simulation in the loop representation (symbols with error bars) to the exact result from Fourier transformation (curves). R.h.s.: Same as on the 1.h.s., now for the chiral susceptibility $C_{\chi}$.

where the number $n_{0}$ is the total number of empty lattice sites. These representations were obtained by differentiating the partition function (2.7).

In Fig. 2 we compare the loop results at $g=0$ (symbols) with those from Fourier transformation (curves) which is applicable in standard representation for the special case of vanishing coupling. Two volumes are used, the relatively small lattice $32 \times 32$ and a substantially larger lattice of $512 \times 512$. For each value of $m$ typically 10000 sweeps were used to equilibrate the system and then about 50000 measurements were performed for our observables. These measurements have been separated by 10 sweeps for each color to guarantee sufficient decorrelation. For the calculation of the observables we used Eqs. (3.4), (3.5), and the statistical error was computed with the jackknife method. For the larger lattice we find almost perfect agreement of the results from the loop representation with the analytic results. In [5] we have presented the results from the loop simulation for several values of $g \neq 0$ and we compared these to the outcome of a simulation with traditional techniques. Concerning the performance we find that with comparable amounts of CPU time with the loop representation we can work on volumes which are two orders of magnitude larger than those available with traditional techniques. The cluster approach of [6] enhances this performance further.

\section{Perspectives and limitations of the loop representation}

Having addressed the merits of the loop representation for the Gross-Neveu model, we would like to comment on possible extensions of the loop method, but also discuss the points where we see limits of the method.

We begin this discussion with stressing that, although we so far restricted our numerical simulations to only two flavors, a generalization to the Gross-Neveu model with an arbitrary number of flavors is straightforward with the loop formula given in [2].

Concerning models with relativistic fermions which are coupled via 4-fermi interactions in higher dimensions, one could try to repeat the strategy that led to the loop representation for the $2-d$ models. An essential step in the identification of the loop formalism was the closed result for the traces of the $\gamma$-matrices. While this is a relatively simple problem in $2-\mathrm{d}$, the corresponding 
structures in 4-d are considerably more involved. For an attempt to find such a closed formula in four dimensions see, e.g., [10].

Interesting might also be the case of non-relativistic fermions in $2+1$ dimensions with 4-fermi interaction. For some of these systems a relation to $3-d$ spin models is known $[11,12]$ which might be useful for a numerical simulation.

We finally comment on the applicability of the loop approach to lattice gauge theories beyond the strong coupling limit. We have already mentioned, that we judge the case of non-abelian gauge fields as an elusive goal, due to the non-commutativity of the link variables. For abelian gauge fields the situation is simpler and in 2-dimensions the loops in (2.6) can again be computed in closed form. The resulting loop representation for the lattice Schwinger Model [7] is of a different type, however. Since gauge fields are oriented quantities, one has to work with oriented loops, while the loops for a scalar interaction are non-oriented (see Eq. (2.7)). One finds that reverting the orientation of a loop corresponds to complex conjugation of its contribution. This implies, that certain cancellations among loops, which simplify the scalar case, are no longer possible [7]. The loops for the Schwinger Model turn out to be self-intersecting and an extra minus sign appears for each intersection. In a numerical simulation [13] it was found that the resulting fermion sign problem limits the size of the accessible volumes. At the moment it is unclear whether this is a fundamental obstacle or if this problem can be overcome by different techniques.

Acknowledgments: We thank Erek Bilgici, Philipp Huber, Christian Lang, Klaus Richter, Andreas Schäfer and Erhard Seiler for discussions and helpful remarks. This work was supported by Fonds zur Förderung der wissenschaftlichen Forschung in Österreich (FWF DK W1203-N08). The simulations were done at the ZID cluster of the Karl-Franzens Unversity Graz.

\section{References}

[1] S. Chandrasekharan and U. J. Wiese, Phys. Rev. Lett. 83 (1999) 3116.

[2] C. Gattringer, Nucl. Phys. B 543 (1999) 533 [arXiv:hep-lat/9811014].

[3] C. Gattringer, Int. J. Mod. Phys. A 14 (1999) 4853 [arXiv:cond-mat/9811139].

[4] D. J. Gross and A. Neveu, Phys. Rev. D10 (1974) 3235.

[5] C. Gattringer, V. Hermann, M. Limmer, Phys. Rev. D 76 (2007) 014503 [arXiv:0704.2277 [hep-lat]].

[6] U. Wolff, arXiv:0707.2872 [hep-lat]; U. Wolff, PoS(LATTICE 2007)284 [arXiv:0710.2179 [hep-lat]].

[7] C. Gattringer, Nucl. Phys. B 559 (1999) 539 [arXiv:hep-lat/9903021].

[8] I.O. Stamatescu, Phys. Rev. D 25 (1981) 1130.

[9] H. Gausterer, C. B. Lang and M. Salmhofer, Nucl. Phys. B388 (1992) 275; H. Gausterer and C. B. Lang, Nucl. Phys. B455 (1995) 785.

[10] K. Scharnhorst, Nucl. Phys. B 581 (2000) 718 [arXiv:hep-lat/0002022].

[11] S. Samuel, J. Math. Phys. 21 (1980) 2820.

[12] C. Gattringer, S. Jaimungal and G.W. Semenoff, Int. J. Mod. Phys. A 14 (1999) 4549 [arXiv:hep-th/9801098].

[13] J. Steiner, Study of the one-Flavour Lattice Schwinger Model with Wilson Fermions in Terms of a 49-Vertex Model, Diploma thesis, University of Graz (2000). 